\title{
ARTICLE
}

\section{The use of Chemometrics to Discriminate Sample Adulteration in Different Levels: the case of Peruvian Maca}

\author{
Heloísa de Carvalho Rodrigues', Hellen Fernanda da Silva Paulino², Patrícia Valderrama1 (iD, \\ Paulo Henrique Março1* iD
}

${ }^{1}$ Programa de Pós-Graduação em Tecnologia de Alimentos, Universidade Tecnológica Federal do Paraná Campus Campo Mourão, Via Rosalina Maria dos Santos, 1233, Área Urbanizada I, 87301-899, Campo Mourão, PR, Brazil

${ }^{2}$ Departamento de Engenharia de Alimentos, Universidade Tecnológica Federal do Paraná - Campus Campo Mourão, Via Rosalina Maria dos Santos, 1233, Área Urbanizada I, 87301-899, Campo Mourão, PR, Brazil

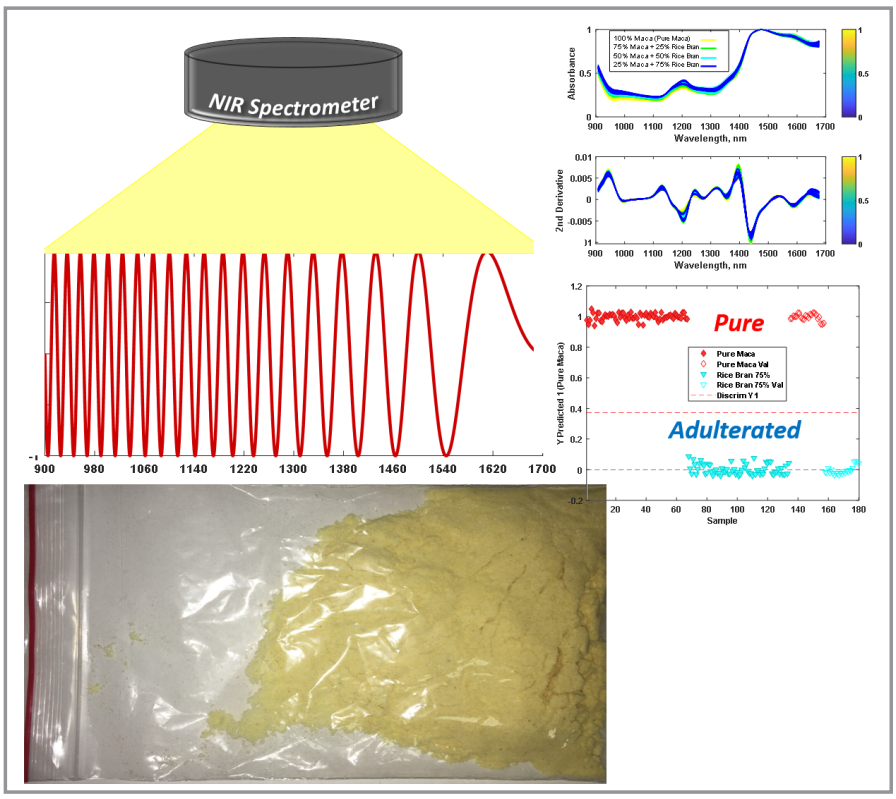

This study aimed to use chemometrics as a tool to allow for near-infrared (NIR) spectroscopy to detect adulteration in Peruvian maca powder. To adulterate the samples, pure maca powder was mixed with rice flour and rice bran, which are the most employed adulterants, using proportions of $25 \%, 50 \%$, and $75 \%$ of each. After adulteration, the mixtures were submitted to NIR spectroscopy. The spectral data were used to build discriminant models through Partial Least Squares Discriminant Analysis (PLS-DA), and each model was evaluated through permutation tests to check for reliability. It was observed that all samples were discriminated against the pure ones, providing unitary sensitivities and specificities. All the models built were tested by the pairwise Wilcoxon signed-rank test, pairwise signed-rank test, and a randomization t-test, and the results indicated that the permuted and un-permuted models were not similar. The results indicated that NIR spectroscopy can be used to discriminate between pure maca powder samples and maca powder adulterated with different levels of rice flour and rice bran, achieving unitary sensitivity and specificity in the PLS-DA models.

Keywords: food analysis, fraud, Peruvian maca, chemometrics, PLS-DA.

Cite: Rodrigues, H. C.; Paulino, H. F. S.; Valderrama, P.; Março, P. H. The use of Chemometrics to Discriminate Sample Adulteration in Different Levels: the case of Peruvian Maca. Braz. J. Anal. Chem., 2021, 8 (32), pp 107-115. doi: http://dx.doi. org/10.30744/brjac.2179-3425.AR-12-2021

Submitted 21 January 2021, Resubmitted 7 April 2021, $2^{\text {nd }}$ time Resubmitted 16 April 2021, Accepted 20 April 2021 , Available online 04 May 2021. 


\section{INTRODUCTION}

As a consequence of the modernization of analytical techniques, in the food industry, the interpretation of the results obtained by chemical analysis referring to the composition of food increasingly demand chemometric strategies, to allow more assertive interventions. Food fraud and adulteration generally aim to increase profit, distorting the desired or required characteristics of the original product. These infractions are recurrent, often due to deficiency or lack of knowledge and or an inspection by the competent authorities. One of the reasons for that is due to the inspection for fraud detection which requires, in most cases, time-consuming analysis and/or expensive techniques, such as the ones derived from chromatography, often demanding sample preparation and trained operator. In this sense, food analysis is a field in which analytical chemistry is mandatory to allow for food authentication.

According to the Food and Agriculture Organization of the United Nations, the term "superfoods" was introduced in 2005 by the food and beverage industry to describe a range of fruit and vegetables thought to confer significant and essential components to human health and nutrition [1]. Nonetheless, there is a need to check not only the active substances which are present in it but also to verify the possibility of adulteration. One of the considered super-foods is the Peruvian maca (Lepidium meyenii), which is a species of tuber grown mainly in the high Andes region of Peru. The consumption of this cultivar is widespread among inhabitants of that region and is usually related to benefits for human health, with the effects attributed mainly to the content of the hypocotyl, the region of the plant where many of the most desired nutrients are concentrated [2]. Interest in maca has been increasing in recent years due to popular appeal related mainly to fertility. However, considering the form in which this plant is legally marketed (flour), makes it susceptible to adulteration by the addition of other flours of similar appearance, which is mainly due to the high market value [3].

Spectroscopic techniques such as in the ultraviolet (UV), near-infrared (NIR), mid-infrared (MIR) regions appear as possible alternatives for industrial processes that can lead to the detection of fraud, bringing advantages such as the analysis speed, little or no sample preparation, non-invasive and non-destructive characteristics, resulting in reduced costs compared to other consolidated techniques, that are used in standard analytical protocols, as shown in earlier studies as in the case of oil capsules [4], that uses to be evaluated by gas-chromatography [5]. However, the use of spectroscopic techniques is challenging when dealing with samples that produce signals with a high degree of similarity, being necessary to use multivariate statistical methods associated with the chemical knowledge of the samples to allow for a feasible interpretation [6].

Among these multivariate options, the partial least squares discriminant analysis (PLS-DA) [7] is a supervised pattern recognition method used for discrimination based on the assignment of classes to known data and has been successfully used for food analysis. In a previous study, we did apply PLS-DA to discriminate lettuce cultivated with and without pesticides evaluated through mass spectrometry [8], to discriminate roasted arabica coffee evaluated by NMR spectroscopy [9], to check for milk adulteration [10] beside other applications. Therefore, this study aimed to discriminate samples of Peruvian maca powder adulterated by adding different proportions of rice flour and rice bran. As far as we know, in Brazil, the main adulteration in this food is done by adding rice bran due to its organoleptic similarity, even though it has never been formally reported, which encourage us to offer this alternative methodology to be used for authentication purposes.

\section{MATERIALS AND METHODS Samples}

Nine different samples of Peruvian maca were purchased in the trade of Campo Mourão (Paraná State, south of Brazil) and region. The samples were found in the form of finely divided powder (in bulk) and in capsules, totaling 9 different brands. Each sample was divided into 10 different portions containing $10 \mathrm{~g}$ of maca powder, totalizing 90 samples. The acquisition was done during the period from November 2018 to February 2019. Besides the Peruvian maca samples, though for adulteration purposes. 


\section{Sample preparation}

The Peruvian maca was adulterated separately with rice flour and rice bran, choosing proportions considered as low (25\%), half (50\%), and high (75\%) adulteration levels (percentage indicating w/w). These proportions were chosen considering the discriminant capability and the amount of sample available to the study. The weighing was performed using an analytical scale model 2204 Bioscale balance, being samples stored in individual plastic bags, duly identified, and stored under refrigeration to be measured the next day. Considering 9 different pure maca, the option was using 10 replicates of each sample, resulting in 90 pure maca samples, 10 pure rice flour samples, 10 pure rice bran samples, 90 samples adulterated with $25 \%$ rice flour, 90 samples adulterated with $25 \%$ rice bran, 90 samples adulterated with $50 \%$ rice flour, 90 samples adulterated with $50 \%$ rice bran, 90 samples adulterated with $75 \%$ rice flour, and 90 samples adulterated with $75 \%$ rice bran. The granulometry was not standardized to simulate a real "in field" application, in which the models would be able to be used for sample screening regarding adulteration.

\section{Near-Infrared Spectroscopy}

The NIR spectra were acquired using a JDSU MicroNIR $1700^{\circledR}$ spectrophotometer, located in the CAMulti laboratory at the UTFPR - Campus Campo Mourão. The measurements were performed using 20 scans for each spectrum. Samples were removed from the refrigerator two hours before starting the measurements to reach room temperature. From each sample, 10 replicates were produced, with the average spectrum of the 10 measurements used as representative of each sample for all data processing. The measurements were taken with the samples inside plastic packages, being the same procedure used for the calibration of $100 \%$ reflectance (with the reflectance sphere also packed in plastic) and turning off the light source to calibrate the zero reflectance.

\section{Chemometrics}

The chemometric procedures adopted were mainly related to the elimination of noise and irrelevant information. Multiplicative Scatter Correction (MSC), standard normal variation (SNV), and second derivatives were used, and the models obtained from these different treatments were compared in order to allow for the choice of the best ones in terms of sensibility/specificity [7]. To build the models, the KennardStone [11] approach was used to select 67 samples for calibration and 23 samples for validation. The best models were built after mean-centering the spectra and performing MSC to the samples adulterated with rice bran. To the samples adulterated with rice flour, the best models also demanded performing second derivatives. All the models were built through cross-validation using the "venetian blinds" method with 10 splits and 1 sample per split.

After pre-processing, PLS-DA was applied to discriminate pure maca samples from $25 \%$ adulterated with rice flour (model 1). Then, pure maca versus $50 \%$ adulteration with rice flour (model 2 ) and so pure maca vs $75 \%$ adulterated with rice flour (model 3). The same procedure was done to the adulterant rice bran, providing model 4 (pure maca vs $25 \%$ adulteration with rice bran), model 5 (pure maca vs $50 \%$ adulteration with rice bran), and model 6 (pure maca vs $75 \%$ adulteration with rice bran). The spectral data were processed using Matlab R2017 ${ }^{\circledR}$ and PLS Toolbox $7.8^{\circledR}$ provided by EMBRAPA Solos do Rio de Janeiro.

\section{PLS-DA performance}

To check for PLS-DA models' performance, the diagnostic tests used were sensitivity and specificity, which details can be found somewhere else [7]. Moreover, the models were evaluated through permutation tests, which are tests in which the "y-block" is randomly reordered. The permutation tests consisted of verifying the Pairwise Wilcoxon signed rank test, Pairwise signed rank test, and a Randomization t-test according to Souza et al [8]. 


\section{RESULTS AND DISCUSSION}

The models built to discriminate between maca powder from rice bran demanded mean-centering and multiplicative scatter correction, while the models for the discrimination between maca and rice flour demanded, besides mean-centering and multiplicative scatter correction, the use of second derivatives. Otherwise, the models are not able to discriminate between these classes (pure vs contaminated). Table I presents the sensitivity, specificity, Pairwise Wilcoxon signed rank test (Wilcoxon), Pairwise signed rank test (Sign Test), and the Randomization $t$-test (Rand $t$-test) to each achieved model. Model 1 refers to the discrimination between pure maca and maca adulterated with $25 \%$ rice bran; model 2 to the discrimination between pure maca and adulteration with $50 \%$ rice bran, and model 3 to the discrimination between pure maca and the adulterated with $75 \%$ rice bran. Models 4, 5, and 6 refer to the adulteration of $25 \%, 50 \%$, and $75 \%$ with rice flour, respectively.

Table I. Parameters of merit of the discrimination models

\begin{tabular}{ccccccc}
\hline Model & $\begin{array}{c}\text { Latent } \\
\text { Variables }\end{array}$ & Sensitivity & Specificity & Wilcoxon & Sign Test & Rand $t$-test \\
\hline 1 & 2 & 1.00 & 1.00 & 0.000 & 0.000 & 0.005 \\
2 & 2 & 1.00 & 1.00 & 0.000 & 0.000 & 0.005 \\
3 & 2 & 1.00 & 1.00 & 0.000 & 0.000 & 0.005 \\
4 & 4 & 1.00 & 1.00 & 0.000 & 0.000 & 0.005 \\
5 & 4 & 1.00 & 1.00 & 0.000 & 0.000 & 0.005 \\
6 & 4 & 1.00 & 1.00 & 0.000 & 0.000 & 0.005 \\
\hline
\end{tabular}

Figure $1(\mathrm{~A})$ presents the spectra of pure maca adulterated with $25 \%, 50 \%$, and $75 \%$ of rice bran, and $(B)$ its respective derivatives, while Figure $1(C)$ presents the spectra of pure maca adulterated with $25 \%, 50 \%$ and $75 \%$ of rice flour and (D) its respective derivatives. It can be observed that the spectra are influenced by the adulteration, increasing vibration intensities below $1400 \mathrm{~nm}$ when adulterated with rice bran and decreasing it when adulteration is made by adding rice flour. Moreover, when the adulteration is done by adding rice bran, it is not possible to see any trend in wavelengths higher than $1500 \mathrm{~nm}$, while for rice flour adulteration the absorbance to this region trends to go down. The rice bran is made from the rice skin (obtained from the polishing of the pealed rice) and rice flour is produced by grinding the rice grains. Therefore, besides the difference in the composition, they differ in the particle size, which is bigger in rice flour than in rice bran. 

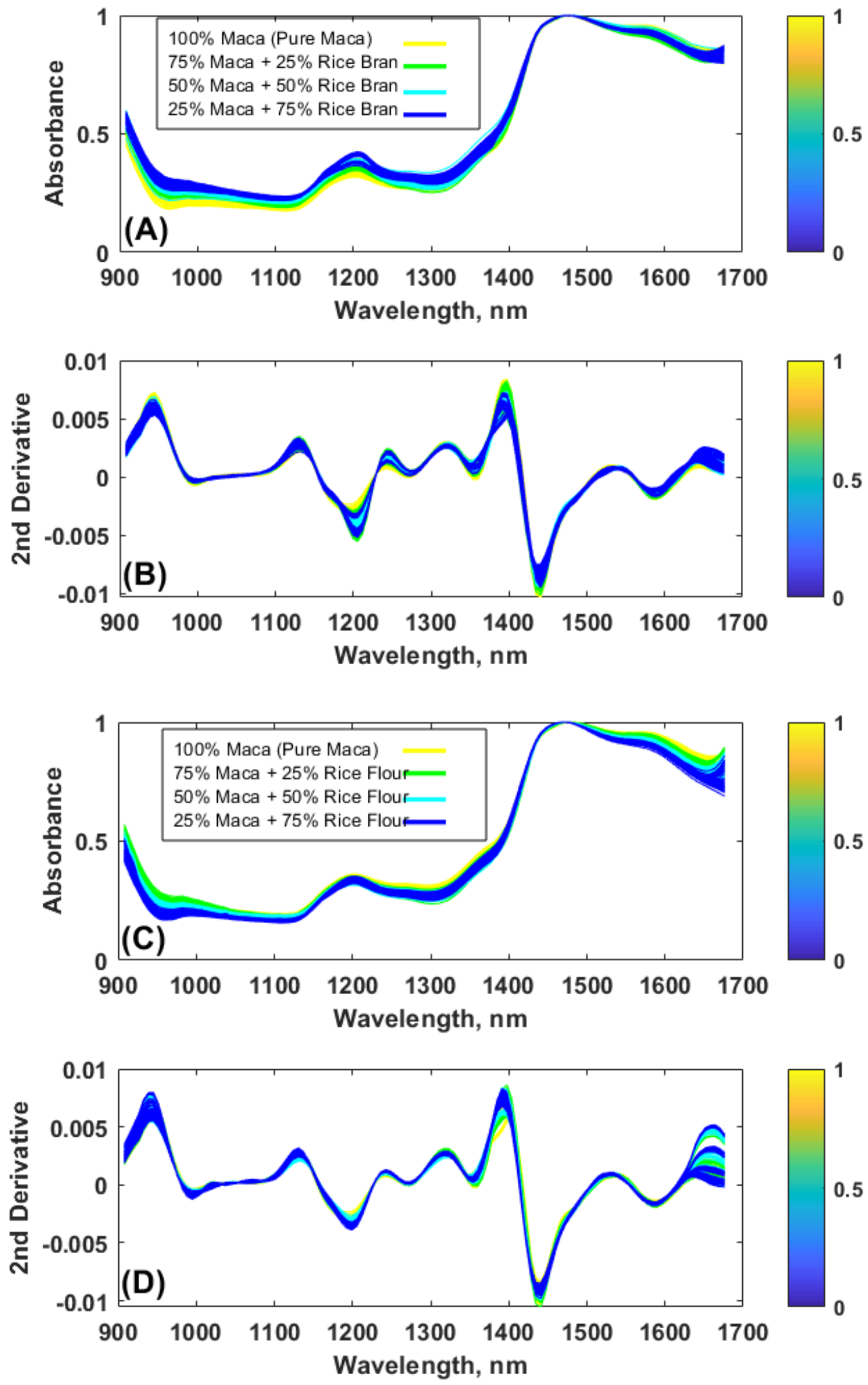

Figure 1. (A) Spectra of the pure maca and respective adulteration with rice bran and (B) its respective derivatives; (C) Spectra of the pure maca and respective adulteration with rice flour and (D) its respective derivatives. 
Figure 2 presents the model's discrimination considering only class 1 (pure maca), to differentiate pure from the adulterated with different bran proportions and the respective VIP scores. The VIP scores indicate that the most important region to be verified is located around 1100-1200 nm, which is assigned to second overtones of methylenic stretches [12]. The regions of $960 \mathrm{~nm}, 1140 \mathrm{~nm}$ and $1200 \mathrm{~nm}$ correspond to $\mathrm{OH}$, $\mathrm{R}-\mathrm{NH}_{2}$, and $\mathrm{CH}$, and are highly prevalent in the protein molecule and can be related to the protein content in the samples [13]. Even though the region around $1400 \mathrm{~nm}$, which is assigned to second combination bands of $\mathrm{OH}$ and $\mathrm{CH}$ bonds, is down the importance limit in the VIP scores (below 1), it is remarkable that this region increases together with the addition of rice bran.
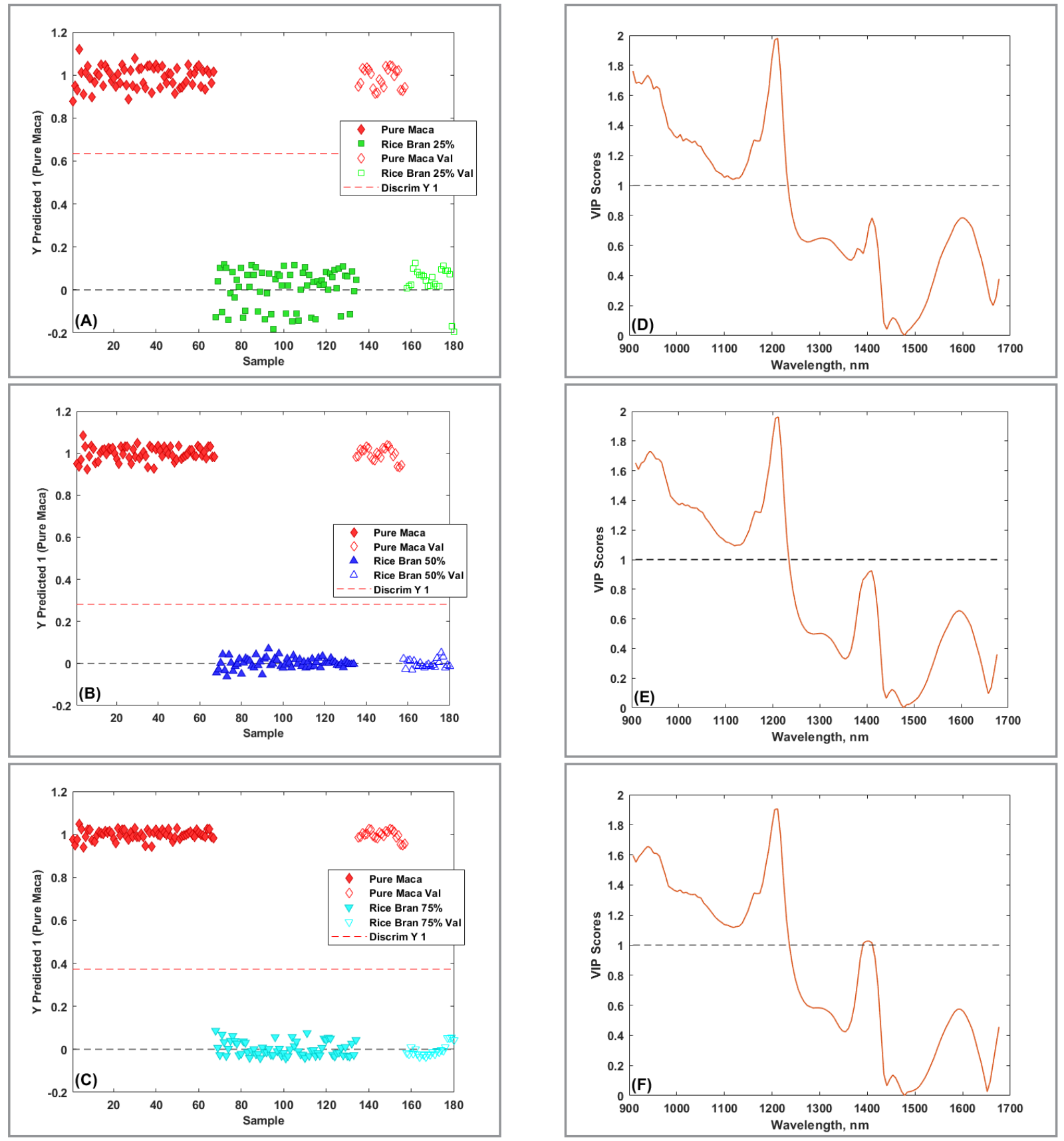

Figure 2. Estimated class values for training and prediction sets for discrimination between (A) pure maca and maca adulterated with $25 \%$ rice bran; (B) pure and adulterated with $50 \%$ rice bran; (C) pure and adulterated with $75 \%$ rice bran. (D), (E) and (F) are the respective VIP Scores. 
The same strategy was used to discriminate between samples adulterated with rice flour, as shown in Figure 3. Looking at the VIP scores, it can be observed that, besides $1200 \mathrm{~nm}$, the region between 1400 $-1500 \mathrm{~nm}$ is above the threshold (value 1), being also important for this discrimination. This region is assigned to the first overtones of $\mathrm{OH}$ stretches [12].

Comparing these effects, the VIP scores suggest that the adulteration made by rice flour is more susceptible to effects attributed to $\mathrm{OH}$ stretches, being possibly related to water absorption, while rice bran is richer in proteins compared to rice flour. Considering the rice bran composition, the protein content varies from $13-18 \%[14,15]$, while to rice flour, this quantity falls to about $4-7 \%$ [16].
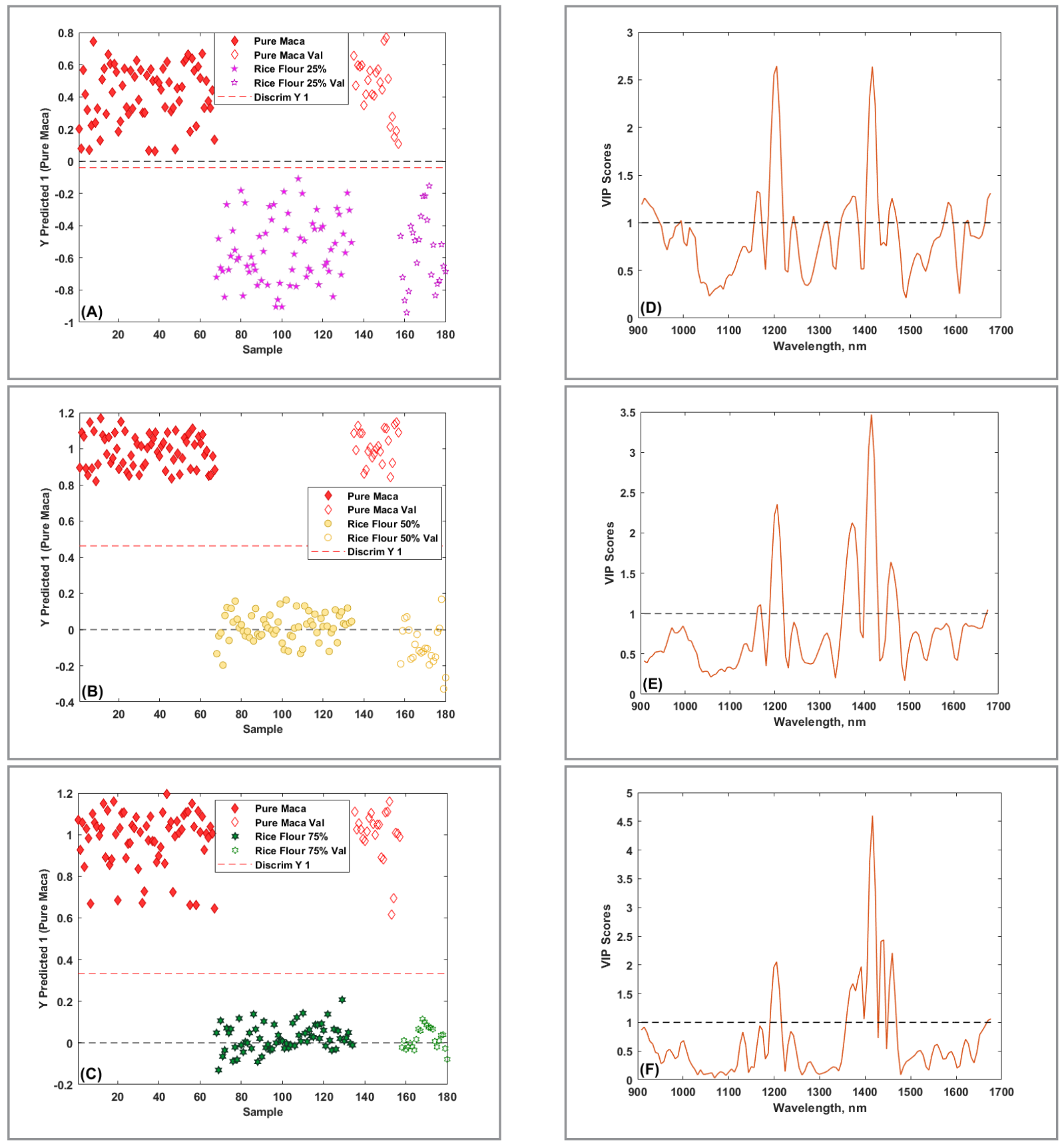

Figure 3. Estimated class values for training and prediction sets for discrimination between (A) pure maca and maca adulterated with $25 \%$ rice flour; (B) pure and adulterated with $50 \%$ rice flour; (C) pure and adulterated with $75 \%$ rice flour. (D), (E) and (F) are the respective VIP Scores. 
The main drawback of using NIR spectroscopy to discriminate samples regarding adulteration is the difficulty to characterize the substances responsible for the differentiation. Nonetheless, it seems to be a feasible tool for sample discrimination, which could allow for a significant increase in the number of evaluated samples, improving the quality control on these emerging foods.

\section{CONCLUSIONS}

NIR spectroscopy can be used as a technique to discriminate Peruvian maca adulteration with rice bran and rice four since it could be associated with chemometrics tools, showing potential to be used as a feasible method for sample discrimination regarding adulteration. The use of PLS-DA on the NIR spectral data allowed for the exploration of different levels of adulteration and the permutation tests confirmed that the models achieved discrimination of maca samples. Moreover, the adulterants did impose different influences on the spectra, increasing the absorbance below $1400 \mathrm{~nm}$ when maca was adulterated with rice bran and decreasing it when adulteration was made by adding rice flour. Also, even though all the models achieved unitary sensibility and specificity, it would be required to increase the variability of the samples to reach more robustness.

\section{Acknowledgements}

This work was supported by the Brazilian National Council for Scientific Research (CNPq), [grant number 485640/2013-9 and Scientific Beginner], and CAPES (001), besides the CAMult-CM laboratories.

\section{Conflicts of interest}

The authors declare that this study has no relationships which may constitute a conflict of interest at any level.

\section{REFERENCES}

1. Hewett, W. E. Post-harvest requirements: farm to market and is there a case for certifying superfruit? In: Proceedings of the International Symposium on Superfruits: Myth or Truth? Ho Chi Minh, Vietnam, on 1-3 July 2013, p 140.

2. Lozano-Canales, A.; Janampa-Santome, M.; Clark, D.; Gonzáles, W. L. Sci. Hortic., 2019, 253, pp 341-348 (https://doi.org/https://doi.org/10.1016/j.scienta.2019.04.059).

3. Beharry, S.; Heinrich, M. J. Ethnopharmacol., 2018, 211, pp 126-170 (https://doi.org/10.1016/j. jep.2017.08.003)

4. Leme, L. M.; Nakamura, F.; Tanamati, A. A. C.; Valderrama, P.; Março, P. H. LWT, 2019, 109, pp 179185 (https://doi.org/10.1016/j.lwt.2019.03.088).

5. Martinez, N.; McDonald, B. Veterinary Dermatology, 2021 (https://doi.org/https://doi.org/10.1111/ vde.12950)

6. Ferreira, M. M. C. Quimiometria: Conceitos, Métodos e Aplicações (ISBN: 9788526810631) Editora da UNICAMP, Campinas, SP, 2015.

7. Brereton, R. G.; Lloyd, G. R. J. Chemom., 2014, 28, pp 213-225 (https://doi.org/10.1002/cem.2609).

8. Souza, S. J.; Valderrama, P.; Consolin Filho, N.; Pilau, E. J.; Tanamati, A. A. C.; Wentzell, P. D.; Março, P. H. J. Chemom., 2020, 34 (12), e3299 (https://doi.org/10.1002/cem.3299).

9. Happyana, N.; Hermawati, E.; Syah, Y. M.; Hakim, E. H. H. Current Research in Nutrition Food Science, 2020, 8 (2), pp 479-488 (https://doi.org/10.12944/CRNFSJ.8.2.13).

10. Mabood, F.; Jabeen, F.; Ahmed, M.; Hussain, J.; Al Mashaykhi, S. A. A.; Al Rubaiey, Z. M. A.; Farooq, S.; Boqué, R.; Ali, L.; Hussain, Z.; et al. Food Chem., 2017, 221, pp 746-750 (https://doi.org/10.1016/j. foodchem.2016.11.109).

11. Kennard, R. W.; Stone, L. A. Technometrics, 1969, 11, pp 137-148 (https://doi.org/10.2307/1266770).

12. Burns, D. A.; Ciurczak, E. W. (Editors) Handbook of near-infrared analysis, $3^{\text {rd }}$ Edition. (ISBN 9780849373930) CRC Press, 2007. 
13. Workman Jr, J.; Weyer, L. Practical Guide and Spectral Atlas for Interpretive Near-infrared Spectroscopy, $2^{\text {nd }}$ Edition. CRC Press, Boca Raton, FL, 2012.

14. Faria, S. A. S. C.; Bassinello, P. Z.; Penteado, M. V. C. Braz. J. Pharm. Sci., 2012, 48 (4), pp 651-657 (https://doi.org/10.1590/S1984-82502012000400008).

15. Liu, Y.; Zhang, H.; Yi, C.; Quan, K.; Lin, B. Food Chem., 2021, 342, 128352 (https://doi.org/10.1016/j. foodchem.2020.128352).

16. He, Y.; Chen, F.; Shi, Y.; Guan, Z.; Zhang, N.; Campanella, O. H. Food Science and Human Wellness, 2021, 10 (1), pp 45-53 (https://doi.org/10.1016/j.fshw.2020.05.010). 